\title{
Face Recognition Using Gabor features And PCA
}

\author{
${ }^{1}$ Miss. Rupali mhaske, ${ }^{2}$ Miss. Jyoti Bedre, ${ }^{3}$ Ms. Shubhangi Sapkal \\ ${ }^{1}$ (Computer science \& Engg. Department, RMCOE/JNT University, India) \\ ${ }^{2}$ (Computer science \& Engg. Department, Govt. College/ Aurangabad University, India)
}

\begin{abstract}
Face recognition is a hot research topic in the fields of pattern recognition and computer vision, which has been found a widely used in many applications, such as verification of credit card, security access control, and human computer interface. As a result, numerous face recognition algorithms have been proposed, and surveys in this area can be found. Although many approaches for face recognition have been proposed in the past, none of them can overcome the main problem of lighting, pose and orientation. For a real time face recognition system, these constraints are to be a major Analysis (PCA). These methods challenge which has to be addressed. In this proposed system, a methodology is given for improving the robustness of a face recognition system based on two well-known statistical modelling methods to represent a face image: Principal Component extract the discriminates features from the face. Preprocessing of human face image is done using Gabor wavelets which eliminates the variations due to pose, lighting and features to some extent.

PCA extract low dimensional and discriminating feature vectors and these feature vectors were used for classification. The classification stage uses nearest neighbour as classifier. This proposed system will use the YALE face data base with 100 frontal images corresponding to10 different subjects of variable illumination and facial expressions.
\end{abstract}

Keywords: Face recognition, Gabor Wavelet transform, Principal Component Analysis

\section{INTRODUCTION}

Face recognition has been a very popular research topic in recent years. The first attempts began in the 1960 's with a semi-automated system. It used features such as eyes, ears, noses, and mouths. Then distances and ratios were computed from these marks to a common reference point and compared to reference data. In the early 1970's Goldstein, Harmon and Lesk created a system of 21 subjective markers such as hair color and lip thickness. Later Kohonen demonstrated that a simple neural net could perform face recognition for aligned and normalized face images. The type of network he employed computed a face description by approximating the eigenvectors of the face image's autocorrelation matrix; these eigenvectors are now known as 'Eigenfaces'. Kohonen's system was not a practical success, however, because of the need for precise alignment and normalization [1]. Turk and Pentland (1991) then demonstrated that when we perform the coding using the eigenfaces the residual error could be used both to detect faces in cluttered natural imagery, and to determine the precise location and scale of faces in an image.

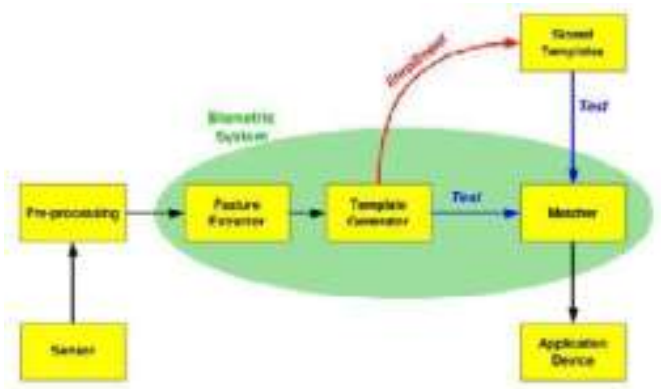

Fig. 1 Block Diagram of Face Recognition

An overview of the face recognition process is illustrated in Fig. 1. In the figure the gallery is the set of known individuals. The images used to test the algorithms are called probes. A probe is either a new image of individual in the gallery or an image of an individual not in the gallery. To compute performance, one needs both a gallery and probe set. The probes are presented to an algorithm, and the algorithm returns the best match between the each probe and images in the gallery. The estimated identity of a probe is the best match.

While there are many databases in use currently, the choice of an appropriate database to be used should be made based on the task given [2]. Some face data sets that are commonly used: Color FERET Database, Yale Face Database, and PIE Database, FIA video Database, CBCL Face recognition Database, 
Expression Image Database, Mugshot Identification Database, Indian Face Database, Face Recognition Data, and University of Essex, UK.

\section{GABOR WAVELETS}

In face recognition system the feature based method finds the important features on the face and represents them in an efficient way. Physiological studies found that the cells in the human virtual cortex can be selectively tuned to orientation and to spatial frequency. This confirmation that the response of the simple cell could be approximated using 2D gabor filters is given by G.Daugmann [3]. Gabor filters were introduced in image processing because of their biological relevance and computational properties $[4,5]$. The kernels of gabor wavelets are similar to $2 \mathrm{D}$ receptive field profiles of the mammalian cortical simple cells. These kernels exhibit desirable characteristics of orientation selectivity and spatial locality. The extraction of local features in an image can be effectively done using gabor wavelets.Using Gabor wavelets is robust to illumination, poses and facial expression changes. Considering all Gabor kernels, all the features are concatenated to form a single gabor feature vector. Then this high dimensional gabor vector space is much reduced by applying statistical modeling methods first PCA and then LDA to obtain more independent and discriminating features.

\section{PRINCIPALCOMPONENT ANALYSIS}

In image recognition and compression, Principal Component Analysis (PCA) is one of the most successful techniques. The large dimensionality of the data space (observed variables) is reduced to the smaller intrinsic dimensionality of feature space (independent variables). This is the case when there is a strong correlation between observed variables and main purpose of using PCA. Using PCA it is capable of transforming each original image of the training set into a corresponding eigenface. The reconstruction of any original image from the training setby combining the eigenfaces is an important feature of PCA which are nothing but characteristic features of the faces. Consequently by adding up all the eigenfaces in the right proportion the original face image can be reconstructed. Each eigenface represents only certain features of the face, which may or may not be present in the original image. If the particular feature is not present in the original image, then the corresponding eigenface should contribute a smaller part to the sum of eigenfaces. If, by contrary, the feature is present in the original image to a higher degree; the share of the corresponding eigenface in the "sum" of the eigenfaces should be greater. So, the reconstructed original image is equal to a sum of all eigenfaces, with each eigenface having a certain weight and this weight specifies, to what degree the specific feature (eigenface) is present in the original image .. That is in order to reconstruct the original image from the eigenfaces, building a kind of weighted sum of all eigenfaces is required. By using all the eigenfaces extracted from original images, exact reconstruction of the original images is possible. by choosing only the most important features (eigenfaces) losses due to omitting some of the eigenfaces can be minimized. Suppose there are $\mathrm{C}$ classes in the training data. PCA is based on the sample covariance which characterizes the scatter of the entire data set, irrespective of class-membership. The projection axes chosen by PCA might not provide good discrimination power.

The algorithm used for principal component analysis is as follows:

\section{1: Mathematics of PCA}

A 2-D facial image can be represented as 1-D vector byconcatenating each row (or column) into a single column(or row) vector.

1) We assume the training sets of images are $\Gamma_{\mathrm{l}}, \Gamma_{2}, \Gamma_{3} \ldots \Gamma_{\mathrm{m}}$, with each image $\mathrm{I}(\mathrm{x}, \mathrm{y})$. Where $(\mathrm{x}, \mathrm{y})$ is the sizeof the image. Convert each image into set of vectors andnew full-size matrix (mxp), where $m$ is the number oftraining images and $\mathrm{p}$ is $x \times y$ the size of the image.

2) Find the mean face by:

$\Psi=\sum \mathrm{I}(\mathrm{x}, \mathrm{y} / \mathrm{m} ; \mathrm{L}: \mathrm{i}=1, \ldots, \mathrm{ri}$.

3) Calculated the mean-subtracted face:

$<1>,=\mathrm{r},-$ 'P

$\mathrm{i}=1,2,3 \ldots \mathrm{m}$. and a set of matrix is obtained with

$\mathrm{A}=[<1>1,\langle 1>2, \ldots \ldots .<1>\mathrm{m}$, $]$ is the mean-subtracted matrixvector with its size Amp.

4) By implementing the matrix transformations, thevector matrix is reduced by:

$\mathrm{Cmm}=\mathrm{Amp} \mathrm{X}$ ATpm (9)where $\mathrm{C}$ is the covariance matrix and T is transposematrix.

5) Find the eigen vectors Vmm and eigen values Amfrom the $\mathrm{C}$ matrix and ordered the eigen vectors byhighest eigen values.

6) Apply the eigen vector matrix, Vmm and adjusted matrix Dm. These vectors determine the linearcombinations of the training set images to form the eigen faces

7) Instead of using $\mathrm{m}$ eigen faces, $\mathrm{m}$ '« $\mathrm{m}$ which isconsidered as the image provided for training for eachindividual or $\mathrm{m}$ ' is the total class used for training.

8) Based on the eigen faces, each image has its facevector by 
$\mathrm{Wk}=\mathrm{u}\left(\mathrm{r}-\mathrm{r}^{\prime}\right), \mathrm{k}=1,2, \ldots, \mathrm{m}$ '.

9) The weights form a feature vector.This feature vectors are taken as the representational basis for the face images with dimension reduced with $\mathrm{m}$.

\section{RESULTS AND DISCUSSION}

In this system is tested with YALE face databaseand its effectiveness is shown in results. The extractedfeatures is used as it is using the above said featureextraction methods and classification is done using eigenvaluesand compared with Euclidean distance measure method.The recognition rate is obtained with fixed number ofPCA features. The performance comparison withEuclidean Distance Measure Classifier (ED) is shown inTable I.

Tablei. Performance Comparison with Ed Classifier

\begin{tabular}{|c|c|c|}
\hline \multicolumn{3}{|c|}{ Euclidean Distance Measure(ED) Classifier } \\
\hline No. of Features & PCA & GABOR + PCA \\
\hline 40 & 85.0 & 89.2 \\
\hline 50 & 82.0 & 88.5 \\
\hline 60 & 92.5 & 94.9 \\
\hline 70 & 93.2 & 98.6 \\
\hline
\end{tabular}

Then using graph, the results analysis can be shown below in figure 2 .

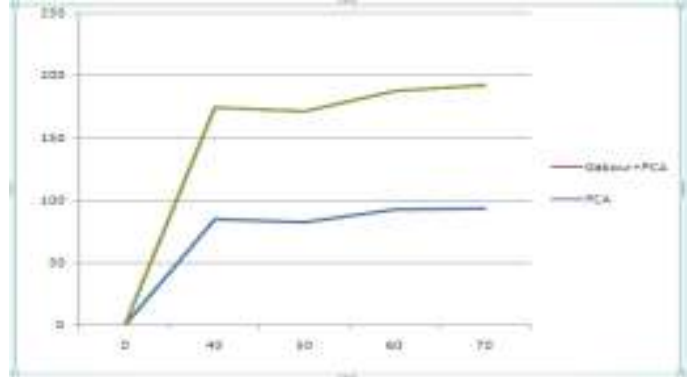

Figure 2. Performance comparison with ED classifier

\section{CONCLUSION}

In this system, a Gabor feature based method used to eliminate the variations due to pose, lighting and features is used to increase the robustness of the face recognition system. For different scales and orientations of the Gabor filter, the input image is convoluted with gabor filters. Then this convoluted image feature vectors were formulated using PCA method. PCA is used to reduce the high dimensionality of these feature vectors. This discriminating feature space is used as the training feature space for Back propagation Neural Network in the classification stage. The recognition rate high with features extracted from PCA based Gabor methods than simple PCA methods. As the number of features selected in PCA increased, this consecutively also increases the recognition rate. But this in turn increases the computational load.

\section{REFERENCES}

[1] Kobonen's system, T. Kobonen, "Self- organization and Associative Memory", Springer-Verlag, Berlin, 1989.

[2] M. Turk and A. Pentland, "Eigenfaces for recognition ", J Cog. Neuroscience, vol. 3, no. I, pp. 71-86, 1991.

[3] Maxim A. Grodin, "Elastic graph matching (EGM) ", On internal representation in face recognition systems, Pattern recognition, Volume 33, Issue 7, July 2000, Page 1161-1177.

[4] Cooley, W. W. and Lohnes, "PCA correlation", P. R. Multivariate Data Analysis John Wiley \& Sons, Inc., New York, 1971).

[5] Thomson, N., Boulgouris, N. V., \& Strintzis, M.G. (2006, January)peak signal-to-noise ratio (PSNR),. Optimized Transmission of JPEG2000 Streams Over Wireless Channels. IEEE Transactions on Image Processing, 15 (I).

[6] W. Zaho, R.Chellappa, P.J.Philips and A.Rosenfeld, "Face recognition A literature survey," ACM Computing Surveys, Vol. 35, No. 4, pp. 399- 458, December 2003.

[7] M. Turk and A. Pentland, "Eigenfaces for recognition," J. Cognitive Neuroscience,vol. 3, 71- 86., 1991

[8] Chen, L.F., M.L. Hong-Yuan, K. Ming-Tat, L. JaChen and Y. Gwo-Jong, "A new LDAbased face recognitionsystem which can solve the small sample size problem", Pattern Recognition, vol 33, pp 1713-1726, 2000.

[9] Vitomir Struc, Nikola pavesi, "Gabor-Based KernelPartial-Least-Squares Discrimination Features for Face Recognition", Informatica, vol. 20, No. 1, 115-138, 2009.

[10] S. Rajasekaran \& G.A. Vijayalakshmi Pai, "Neural Networks, Fuzzy Logic and Genetic Algorithms" Prentice-Hall of India Private Limited, 2003.

[11] T.Yahagi and H.Takano,(1994) "Face Recognition using neural networks with multiple combinations of Categories," International Journal of Electronics Information and Communication Engineering., vol.J77-D-II, no.11, pp.2151-2159. 